\title{
Infección por Chlamydia trachomatis en usuarias de dos clínicas de planificación familiar
}

Thelma Canto-de Cetina, MC, ${ }^{(1)}$ Lucila Polanco-Reyes, MC, ${ }^{(1)}$

Víctor Fernández-G onzález, Q FB, ${ }^{(1)}$ Sandra Ruiz-García, Lic en Enf. ${ }^{(1)}$

\begin{abstract}
Canto-de CetinaT, Polanco-Reyes L, Fernández-González V, Ruiz-García S. Infección por Chlamydia trachomatis en usuarias de dos clínicas de planificación familiar. Salud Publica Mex 2003;45 supl 5:S657-S661. El texto completo en inglés de este artículo está disponible en: http://www.insp.mx/salud/index.html
\end{abstract}

\section{Resumen}

0 bjetivo. D eterminar la prevalencia de infección por C hlamydia trachomatis en mujeres que acuden a dos clínicas de planificación familiar. Material y métodos. Se llevó a cabo un estudio transversal en 1100 mujeres sexualmente activas, aparentemente sanas, de Mérida, Yucatán, México, quienes acudieron a dos clínicas de planificación familiar en el periodo comprendido de enero a diciembre de 1998. Las mujeres incluidas en el estudio tuvieron entre 15 y 45 años de edad, y habían estado libres de tratamiento antibacteriano al menos durante el mes previo a su inclusión. Se obtuvieron muestras endocervicales y datos epidemiológicos. La detección de bacterias fue realizada por el método inmunoenzimático de marcaW ellcozyme. Los datos fueron procesados en el programa EPI Info. Como métodos estadísticos se utilizaron la prueba de Z, ji cuadrada y como medida de asociación, la razón de prevalencias. Resultados. Setenta y cuatro mujeres $(6.7 \%)$ fueron positivas a infección por Chlamydia trachomatis. Cerca de $50 \%$ de las mujeres estuvo asintomática. No se encontró diferencia estadística entre la proporción de mujeres con y sin Chlamydia que tuvieron síntomas vulvovaginales; por el contrario, los cambios relacionados con cervicitis fueron más frecuentes en las pacientes infectadas. C trachomatis fue más frecuente en usuarias de anticonceptivos or ales (8.3\%) comparadas con mujeres que no tenían método anti-

\author{
Canto-de CetinaT, Polanco-Reyes L, \\ Fernández-González V, Ruiz-García S. \\ Chlamydia trachomatis infection \\ in users of two family planning clinics. \\ Salud Publica Mex 2003;45 suppl 5:S657-S661. \\ The English version of this paper \\ is available at: http://www.insp.mx/salud/index.html
}

\section{A bstract}

0 bjective. To determine the prevalence of $\mathrm{C}$ hlamydia trachomatis in women attending two family planning clinics in Merida,Yucatan, Mexico. Material and M ethods. From January to December 1998, a cross-sectional study was conducted in 1100 sexually active women between 15 and 45 years of age. Study subjects had not received antibiotic therapy for at least one month prior to their visit to the clinic. Endocervical samples were taken for bacterial detection with the enzymaticW ellcozyme immuno assay method. D ata were analyzed using the Epi-Info program. Statistical analysis was conducted using the chi-squared test and prevalence ratios. Results. Seventy four women were positive to Chlamydia trachomatis $(6.7 \%)$. Almost $50 \%$ of those women were asymptomatic. No statistically significant differences were found between the group with Chlamydia and the group without it who had vulvar-vaginal symptoms. Cervical changes were more frequent in infected women. More positive cases of Chlamydia infection were found in oral contraceptive users (8.3\%) than among non users (5.4\%). Conclusions. Study results confirm the high prevalence of asymptomatic infections and emphasize the importance of timely diagnosis to avoid infection sequelae. The English version of this paper is available at:http://www.insp.mx/salud/ index.html

Estos resultados fueron publicados parcialmente en la revista de divulgación: Inf Infecy Micro 2000:21(3);102-105.

Los resultados son parte de una investigación financiada por el Sistema Regional de Investigación "Justo Sierra Méndez" (C onacyt)(Sisierra) Clave 950204.

(1) Laboratorio de Biología de la Reproducción del Centro de Investigaciones Regionales D r. Hideyo N oguchi, Universidad Autónoma deYucatán, Mérida, Yucatán, México.

Fecha de recibido: 9 de julio de 2001 - Fecha de aprobado: 21 de mayo de 2003.

Solicitud de sobretiros: Dra.Thelma C anto-de Cetina. Laboratorio de Biología de la Reproducción del Centro de Investigaciones Regionales D r.Hideyo N oguchi, Universidad Autónoma de Yucatán, México. Avenida Itzaes N 0.490 x 59 Mérida, Yucatán, México.

Correo electrónico: tcetina@ tunku.uady.mx 
conceptivo (5.4\%). Conclusiones. Los resultados de este estudio confirman la alta prevalencia de infecciones asintomáticas y pone énfasis en la importancia de un diagnóstico oportuno para evitar secuelas. El texto completo en inglés de este artículo está disponible en:http://ww w.insp.mx/salud/ index.html

Palabras clave: Chlamydia trachomatis; infección cervicovaginal; prevalencia; México
Key words: Chlamydia trachomatis; cervicovaginal infection; prevalence; Mexico
$L$ a Chlamydia trachomatis(CT) es considerada como el patógeno más importante de los causantes de infecciones de transmisión sexual (ITS) en países desarrollados y en desarrollo, ${ }^{1}$ y constituye la causa bacteriana más frecuente de dichas enfermedades, las cuales típicamente se manifiestan como cervicitis y uretritis. La infección por esta bacteria en las mujeres produce secuelas y complicaciones graves como la enfermedad inflamatoria pélvica, la infertilidad y el embarazo ectópico. ${ }^{2-4}$ Puede producir abortos repetidos, ruptura prematura de membranas y es causa frecuente de bajo peso al nacer, con aumento de la mortalidad perinatal; asimismo, es responsable de conjuntivitis y neumonía en el recién nacido, debido a la infección genital materna. ${ }^{5}$

La prevalencia de CT varía en diversos estudios: en mujeres asintomáticas que asisten a clínicas de planificación familiar se ha reportado entre 3 y $5 \%$, aumentando a cifras mayores de $20 \%$ en las clínicas de ITS. ${ }^{6-7}$ En embarazadas varía entre 2 y 37\%; ${ }^{8-10}$ asimismo, se ha sugerido que las mujeres que toman anticonceptivos orales son más propensas al desarrollo de cervicitis, debido en parte a la presencia de ectropión cervical. ${ }^{11}$

Estudios realizados en México han demostrado una frecuencia de 4 y $28 \%$ en mujeres no embarazadas, consideradas como de bajo riesgo para las ITS; de $16.6 \%$ en trabajadoras sexuales y hasta de 10 a $12 \%$ en embarazadas. ${ }^{12-16}$

Aunque en algunos países existen guías para la prevención y el tratamiento de las infecciones por $\mathrm{CT}$, éstas deben ajustarse para cada uno, por lo que es necesario definir los grupos considerados de alto riesgo para esta infección, para lo cual se requiere el conocimiento del perfil demográfico y la prevalencia local y por grupos de riesgo, con el objeto de optimizar los programas de detección.

En México no se conocen la prevalencia y el impacto causado por esta infección en diferentes grupos de mujeres, debido a la relativa dificultad para establecer el diagnóstico, ya que la metodología de laboratorio es técnicamente demandante y costosa. Por tal motivo, consideramos importante llevar a cabo el siguiente trabajo de investigación en usuarias de clínicas de planificación familiar de Mérida,Yucatán, México, con el objetivo de establecer la prevalencia de infección por CT en dichas mujeres.

\section{Material y métodos}

El estudio se llevó a cabo en 1100 usuarias, aparentemente sanas, de dos clínicas de planificación familiar, una situada en el Centro de Atención Materno Infantil de la Secretaría de Salud de Mérida (717), y la otra en el Centro de Investigaciones Regionales "Dr. Hideyo Noguchi" (383), de enero a diciembre de 1998. Las pacientes llenaron los siguientes criterios de inclusión: haber aceptado participar en el estudio, edad comprendida entre 15 y 45 años, que fueran sexualmente activas y que no hubieran recibido antibióticos el mes anterior al estudio.

A todas se les aplicó un cuestionario estandarizado que incluyó datos sociodemográficos como edad y estado civil, antecedentes ginecoobstétricos, como la ocurrencia de abortos o muertes fetales durante alguna época de su vida, así como bajo peso del producto al nacer en su último embarazo, conducta sexual y número de parejas. A las mujeres incluidas en el estudio se les practicó examen ginecológico por médico especialmente entrenado, y se consignaron los datos clínicos y ginecológicos, el aspecto de la vagina y del cérvix, existencia de flujo vaginal y de sus características. Para la detección de CT se tomaron especímenes del canal cervical, con espéculo vaginal e hisopo, evitando el contacto con la mucosa vaginal y se remitieron al laboratorio de Biología de la Reproducción del Centro de Investigaciones Regionales para ser procesados por medio de inmunoanálisis enzimático cualitativo, de la marca Wellcozyme Chlamydia, el cual se basa en un sistema de micropozos que utiliza anticuerpos monoclonales contra el liposacárido (LPS) Chlamydial en fase sólida, y un conjugado (anticuerpo monoclonal contra LPS Chlamydial), marcado con 
una enzima(fosfatasa alcalina) de una sensibilidad de $84.4 \%$ y una especificidad de $99.5 \%$, de acuerdo con las especificaciones del fabricante.

Se tomaron en cuenta las siguientes definiciones:

Aborto: pérdida de un producto antes de las 20 semanas de gestación. ${ }^{17}$

Bajo peso al nacer: producto menor de $2.5 \mathrm{~kg} .{ }^{17}$

Parto pretérmino: parto ocurrido antes de las 37 semanas de gestación. ${ }^{17}$

Análisis estadístico: se utilizó la prueba de Z para variables cuantitativas y la ji cuadrada para variables cualitativas, así como la razón de prevalencia para investigar la asociación entre el uso de método anticonceptivo oral con CT; se consideraron como estadísticamente significativos valores de $p<0.05$. Se utilizó el programa Epi Info.

\section{Resultados}

Se estudió un total de 1100 mujeres de las cuales 74 (6.7\%) resultaron con diagnóstico positivo para CT. Se compararon las 74 mujeres con diagnóstico positivo de CT con las otras 1026 participantes y cuyos resultados fueron negativos en relación con algunas características, como puede verse en el cuadro I. No se encontraron diferencias estadísticamente significativas en relación con la media de la edad en el momento de efectuar el estudio $(27.01 \pm 3.9$ y $28.0 \pm 4.1)$ en las mujeres con diagnóstico negativo y positivo, respectivamente; tampoco con la edad de inicio de la actividad sexual, de la menarca, ni del número de parejas sexuales entre ambos grupos; por el contrario, la frecuencia de antecedentes de parto pretérmino (RP 2.31, IC 95\% 1.3-4.12, $\mathrm{p}=0.009$ ) abortos (RP 1.73, IC 95\% 1.09-2.75, $\mathrm{p}=0.03) \mathrm{y}$ bajo peso al nacer (RP 2.03, IC 95\% 1.11-3.7, p=0.03) fueron mayores en las mujeres con diagnóstico positivo de CT que en aquellas en las que no se aisló la bacteria y esta diferencia fue estadísticamente significativa $(\mathrm{p}<0.05)$.

Con respecto al uso de métodos anticonceptivos en el momento de efectuar el estudio 148 mujeres no tenían método de planificación familiar, 578 estaban utilizando anticonceptivos orales de tipo combinado (0.03 mg de etinylestradiol y $0.300 \mathrm{mg}$ de norgestrel); 299 (31.5\%), inyectable mensual (25 mg de acetato de medroxyprogesterona y $5 \mathrm{mg}$ de cipionato de estradiol); 50 (2.2\%), dispositivo tipo Tcu 380 A y 25 (1.1\%) usaban preservativo.

Aunque se encontró una frecuencia mayor de casos positivos por Chlamydia trachomatis estadísticamente significativa en usuarias de anticonceptivos orales, comparadas con las mujeres que no tenían mé- todo anticonceptivo, (RP 1.67, IC 95\% 1.05-2.65, p= 0.03) no se pudo establecer una asociación entre el uso de este método y la frecuencia de infección por CT. Cerca de $50 \%$ de las mujeres con infección por CT no presentaron manifestaciones clínicas.

Como puede verse en el cuadro II, la presencia de flujo vaginal y de prurito vulvar fue similar en ambos

Cuadro I

Características generales de 1100 usuarias

DE UNA CLÍNICA DE PLANIFICACIÓN FAMILIAR CON Y SIN C HLAM YDIA TRACH O MATIS. MéridA, YuCATÁn, MéXICO, 1996-1998

\begin{tabular}{|c|c|c|c|}
\hline & $\begin{array}{c}\text { Negativas } \\
1026\end{array}$ & $\begin{array}{c}\text { Positivas } \\
74\end{array}$ & $p$ \\
\hline Edad & $27.0 \pm 39$ & $28.0 \pm 4.1$ & NS \\
\hline Inicio de vida sexual & $18.8 \pm 2.2$ & $18.1 \pm 1.9$ & NS \\
\hline Menarca & $11.7 \pm 1.5$ & $11.3 \pm 1.3$ & NS \\
\hline Número de embarazos & $3.4 \pm 5.5$ & $3.6 \pm 2.0$ & NS \\
\hline Más de una pareja sexual & $108 \quad 10.5 \%$ & $810.8 \%$ & NS \\
\hline Bajo peso al nacer & $76 \quad 7.4 \%$ & $1114.9 \%$ & $<0.05$ \\
\hline Parto pretérmino & $73 \quad 7.1 \%$ & $1216.2 \%$ & $<0.05$ \\
\hline Abortos & $21520.9 \%$ & $2432.4 \%$ & $<0.05$ \\
\hline
\end{tabular}

Método anticonceptivo

\begin{tabular}{lrrrrc} 
N inguno & 140 & $94.5 \%$ & 8 & $5.4 \%$ & N S \\
\hline O rales & 530 & $91.6 \%$ & 48 & $8.3 \%$ & $<0.05$ \\
\hline Inyectables & 283 & $94.6 \%$ & 16 & $5.3 \%$ & N S \\
\hline DIU & 49 & $98 \%$ & 1 & $2 \%$ & N S \\
\hline Barrera & 24 & $96 \%$ & 1 & $4 \%$ & N S
\end{tabular}

N S: No significativo

Cuadro II

Aspecto del Cérvix uterino en 1100 usuarias CON Y SIN C HLAM YDIA TRACH O MATIS. MÉRIDA, YuCATÁN, MéXıCO, 1996-1998

\begin{tabular}{lrrrrr} 
& $\begin{array}{c}\text { Negativas } \\
1\end{array}$ & $\begin{array}{c}\text { Positivas } \\
74\end{array}$ & $p$ \\
& 492 & $47.9 \%$ & 34 & $45.9 \%$ & N S \\
$\begin{array}{l}\text { Antecedentes de flujo vaginal } \\
\begin{array}{l}\text { Aspecto del cérvix } \\
\text { Limpio }\end{array}\end{array}$ & 319 & $31.0 \%$ & 12 & $16.2 \%$ & $p<0.05$ \\
\hline Enrojecido & 323 & $31.5 \%$ & 6 & $8.1 \%$ & $p<0.05$ \\
\hline Ectropión & 215 & $20.9 \%$ & 33 & $44.6 \%$ & $p<0.05$ \\
\hline Ulcerado & 169 & $16.5 \%$ & 23 & $31.0 \%$ & $p<0.05$ \\
\hline Flujo vaginal & 186 & $18.1 \%$ & 13 & $17.6 \%$ & N S \\
\hline Prurito vulvar & 74 & $7.2 \%$ & 5 & $6.7 \%$ & N S
\end{tabular}


grupos, no así la de alteraciones en el cérvix, ya que tanto el ectropión como la úlcera cervical fueron más frecuentes en el grupo con resultado positivo para CT (diferencia estadísticamente significativa) $(\mathrm{p}=0.010866)$.

\section{Discusión}

Chlamydia trachomatis es la causa más frecuente de infección del tracto genital femenino y uno de los agentes etiológicos más conocidos en la enfermedad inflamatoria pélvica. La presencia de infección endocervical no tratada durante la vida sexual activa representa un alto riesgo de contagio potencial para la pareja sexual y para el producto, en caso de embarazo; se han reportado prevalencias variables de infección por CT, debido a la falta de estandarización de los métodos de diagnóstico de esta enfermedad y a las diferencias socioeconómicas y étnicas de los diferentes grupos estudiados.

Existen varios métodos para la detección de CT: cultivo de tejidos, métodos inmunoenzimáticos, titulación de anticuerpos IgG anti CT y la reacción en cadena de la polimerasa (PCR). ${ }^{18}$ En México se han llevado a cabo varios estudios para establecer la prevalencia de infección cervicovaginal. Así se ha obtenido en mujeres sexualmente activas, con bajo riesgo para la infección, una prevalencia de 4 y de $7 \%$, utilizando el cultivo celular e inmunofluorescencia directa (Kit comercial Syva Microtrack), respectivamente, ${ }^{12,15}$ con esa misma técnica se encontró, en embarazadas, una frecuencia de $10^{16}$ y de $28.4 \%$ en mujeres con diferentes problemas que asistieron a la consulta de ginecología, ${ }^{13}$ también se han utilizado técnicas inmunoenzimáticas en estudios realizados en trabajadoras del sexo comercial consideradas como población de alto riesgo, en quienes se encontró una prevalencia de $16.6 \% .{ }^{14}$ Utilizamos un método diagnóstico similar a este último; como todo método enzimático tiene ventajas como su relativa sencillez en la aplicación y estandarización de la tecnología, ya que no requiere equipo especial, y, principalmente, por su fácil transporte, puesto que las muestras se tomaron en dos clínicas diferentes; sin embargo, tiene como desventaja la de una menor sensibilidad y especificidad que el método de cultivo de células endocervicales.

Con este método encontramos una prevalencia de infección por CT de $6.7 \%$ en usuarias de dos clínicas de planificación familiar (a las que asisten mujeres con características similares: de clase socioeconómica baja, sin seguridad social). Esta prevalencia está en el rango publicado por la literatura mundial, ${ }^{4,6,8,19}$ aunque es un poco más elevada que la reportada en otro estudio realizado en México, en mujeres con bajo riesgo para la adquisición de infecciones de transmisión sexual. ${ }^{12}$

Como podemos notar (cuadro I) no hubo diferencias estadísticamente significativas en la mayoría de las características generales, entre las mujeres que fueron positivas, en comparación con el grupo de negativas, excepto por el antecedente de abortos, partos pretérmino y producto de bajo peso al nacer; datos que ya han sido reportados en otros estudios previos. ${ }^{5,20-26}$

Contrariamente a lo que se ha publicado, no encontramos relación entre la actividad sexual temprana y el número de parejas con la infección, ya que ambas características fueron similares en las mujeres con resultados positivos y negativos.

Por lo que respecta al uso de métodos anticoncetivos, encontramos que las usuarias de $\mathrm{AO}$ tuvieron un porcentaje significativamente más elevado $(\mathrm{p}<0.05)$ de infección por CT, comparadas con las mujeres sin anticoncepción, aunque similar a la reportada en un trabajo previo efectuado en mujeres con ese mismo método. ${ }^{27}$

Encontramos una alta prevalencia de infecciones asintomáticas, ya que únicamente 13 de las mujeres (7.6\%) con CT se quejaron de flujo vaginal, síntoma considerado como el más común en cualquier tipo de infección vaginal; sin embargo, en el examen ginecológico se detectaron cambios en el cérvix en $62(83.8 \%$ ) y de éstos el más frecuente fue la presencia de ectropión, ya que en 33 de las 74 mujeres $(4.6 \%)$ se detectó esta alteración.

Debemos llamar la atención sobre el hecho de que la presencia de CT indica daño y un posible factor de riesgo. La importancia de hacer el diagnóstico y de iniciar el tratamiento adecuadamente y en forma temprana está plenamente justificada, ya que cerca de $50 \%$ de las infecciones son asintomáticas, pueden permanecer latentes por varios años y aproximadamente $8 \%$ de las infecciones no tratadas progresa a salpingitis, ${ }^{5}$ una de las principales causas de obstrucción tubaria y por ende de esterilidad. Se sabe que el riesgo de infertilidad aumenta con el número de infecciones. Asimismo, es fundamental no olvidar dar tratamiento adecuado a la pareja.

\section{Agradecimientos}

Nuestro agradecimiento a las autoridades y al personal médico y paramédico del Hospital Materno Infantil de la Secretaría de Salud de Mérida,Yucatán.

\section{Referencias}


1.W orld Health 0 rganization. Sexually transmited diseases. Press release W H O /64,25 August, 1995.

2. Jones RB,Mammel JB,Shepard MK,Fisher RR. Recovery of Chlamydia trachomatis from the endometrium of women at risk for chlamydial infection. Am J 0 bstet Gynecol 1986;155:35-39.

3. Cates W,W asserheit JN . G enital chlamydial infection:Epidemiology and reproductive sequelae.Am J 0 bstet Gynecol 1991;164:1771-1781. 4. Faro S. C hlamydia trachomatis: Female pelvic infection. Am J 0 bstet Gynecol 1991;164:1767-1770.

5. Mc Gregor J, French Jl. Chlamydia trachomatis infection during pregnancy.Am J O bstet Gynecol 1991;164:1782-1789.

6. 0 riel JD, Johnson AL,Barlow D,Thomas BJ,N ayyar K, Reeve P. Infection of the uterine cervix with Chlamydia trachomatis.J Infect D is 1978;137: 443-451.

7. Magder LS, Harrison HR, Ehret JM, Anderson TS, Judson FN . Factors related to genital $\mathrm{Chlamydia}$ trachomatis and its diagnosis by culture in a sexually transmitted diseases clinic. Am J Epidemiol 1988;128:298-308. 8. Schachter J. Chlamydial infections.N Engl J Med 1978;298:540-547. 9. Sweet RL,Landers DV,W alker C, Schachter J. C hlamydia trachomatis infection and pregnancy outcome.Am J O bstet Gynecol 1987;156: 824-833.

10. Andrews W W, Goldenberg RL,H auth JC.Preterm labor: Emerging role of genital tract infections.Infect A gent D is 1995;4:196-211. 11.W ashington AE, G ove S, Schachter J,Sweet RL.O ral contraceptives, Chlamydia trachomatis infection, and pelvic inflamatory disease.A word of caution about protection.JAMA 1985;253:2246-2250.

12. Echániz-Avilés $G, C$ alderón-Jaimes $E, C$ arnalla-Barajas $N$, SotoN oguerón A, Cruz-Valdez A, G atica-Marquina R. Prevalencia de infección cervicovaginal por Chlamydia trachomatis en población femenina de la ciudad de Cuernavaca, Morelos.Salud Publica Mex 1992;34:301-307. 13. Rosas-Arceo J,Toca-Porraz L, Díaz-Esponda C, N ava-Flores J. Infección por Chlamydia trachomatis en cérvix uterino.G inecol 0 bstet Mex 1993;61:326-328.

14. Alvarado-Esquivel C, G arcía-Villanueva A, C astruita-Limones DE, C ardosa-N evárez FJ, Ruiz-Astorga R. Prevalencia de infección por Chlamydia Trachomatis en prostitutas registradas en la ciudad de Durango, México. Salud Publica Mex 2000;42:43-47.

15. A costa-Cázares B. Chlamydia trachomatis como factor de riesgo de algunas complicaciones del embarazo.Enferm Infecc Microbiol 1996;16: 142-145.
16. Díaz-Barreiro G,Díaz-López E,Servín-Ramírez F. Frecuencia de Chlamydia trachomatis en el cérvix de pacientes embarazadas en control prenatal. Ginecol 0 bstet Mex 1997;65:48-51.

17. C unningham Mc, D onald $\mathrm{G}$ ant, ed. 0 bstetricia de W illiams. 4a ed. Barcelona, España: Ed. Masson, 1996.

18. Black CM. C urrent methods of laboratory diagnosis of Clamydia trachomatis infections.Clin Microbiol Rev 1997;10:160-184.

19. Schachter J,Stoner E,Moncada J.Screening for chlamydial infections in women attending family planning clinics. Evaluation of presumptive indicators for therapy.W est J Med 1983;138:375-379.

20. Martin DH.Prematurity and perinatal mortality in pregnancies complicated by maternal Chlamydia trachomatis infections.JAMA 1982;247:1585-1587.

21. Harrison HR,Alexander ER,W einstein L,Lewis M,N ash M,Sims DA.Cervical Chlamydia trachomatis and mycoplasmal infections in pregnancy: Epidemiology and outcomes. JAMA 1983;250:1721-1727. 22. Lamont RF,Taylor-Robinson D,N ewman M,W igglesworth J,Elder MB.Spontaneous early preterm labour associated with abnormal genital bacterial colonization.Br J O bstet Gynecol 1986;93:804-810.

23. Ismail MA,Moawad A H,Poon E,Henderson C.Role of Chlamydia trachomatis in post-partum endometritis.J Reprod Med 1987;32: 280-284.

24. Martus J.Relationships of vaginal Lactobacillus sp,cervical C hlamydia trachomatis and bacterial vaginosis with preterm birth. 0 bstet $\mathrm{G} y n e c o l$ 1988;71:89-91.

25. Ryan GM ,A bdellaTN ,M CN eeley G,Baselski VS,D rummond DE. Chlamydia trachomatis infection in pregnancy and effect of treatment on outcome.Am J O bstet Gynecol 1990;162:34-39.

26. Andrews W W, Goldenberg RL,Mercer B,Lams J,Meis P,M ouad A et al. The preterm prediction study:Association of second-trimester genitourinary chlamydia infection with subsecuent spontaneous preterm birth.Am J O bstet Gynecol 2000;183:662-668.

27. C anto-de Cetina T, Polanco RL, C upul YD G, Fernándes GV, Piña CR, Ballote ZM. Prevalencia de infección por Chlamydia Trachomatis en usuarias de una clínica de planificación familiar en Mérida,Yucatán, Enf Infec y Micro. 2001;21:102-105. 